\title{
Determination of optimal cut-off points after a high-fat meal corresponding to fasting elevations of triglyceride and remnant cholesterol in Chinese subjects
}

Jin $\mathrm{Xu}^{1,2}$, Yan-Qiao Chen ${ }^{1,2}$, Shui-Ping Zhao ${ }^{1,2}$ and Ling Liu ${ }^{1,2^{*}}$ (1)

\begin{abstract}
Background: Postprandial high triglyceride (HTG), marking elevated level of remnant cholesterol (RC), is an independent risk factor of coronary heart disease (CHD). The postprandial cut-off points for HTG and high RC (HRC) after a daily meal are recommended as $2.0 \mathrm{mmol} / \mathrm{L}$ and $0.9 \mathrm{mmol} / \mathrm{L}$, respectively, by the European Atherosclerosis Society (EAS), while those after a high-fat meal in Chinese subjects were not explored.

Methods: Ninety subjects, including 60 CHD patients (CHD group) and 30 non-CHD controls (CON group), were enrolled in this study. Serum levels of blood lipids, including calculated RC, were monitored at 0, 2, 4 and $6 \mathrm{~h}$ after a high-fat meal with $800 \mathrm{kcal}$ and $50 \mathrm{~g}$ fat. Analysis of c-statistic was used to determine the cut-off points for postprandial HTG and HRC.

Results: Postprandial levels of triglyceride (TG) and RC significantly increased and peaked at $4 \mathrm{~h}$ after a high-fat meal in two groups, although those in CHD group were significantly higher $(P<0.05)$. The optimal cut-off point to predict $\mathrm{HTG}$ at $4 \mathrm{~h}$ corresponding to fasting $\mathrm{TG} \geq 1.7 \mathrm{mmol} / \mathrm{L}$ was $3.12 \mathrm{mmol} / \mathrm{L}$, and that to predict $\mathrm{HRC}$ at $4 \mathrm{~h}$ corresponding to fasting $\mathrm{RC} \geq 0.8 \mathrm{mmol} / \mathrm{L}$ was $1.36 \mathrm{mmol} / \mathrm{L}$. According to the new cut-off points, the omissive diagnosis rates of postprandial HTG and HRC decreased obviously.

Conclusion: The cut-off points of postprandial HTG and HRC in Chinese subjects after a high-fat meal were higher than those after a daily meal recommended by the EAS, indicating that specific cut-off points should be determined after a certain high-fat meal.
\end{abstract}

Keywords: Postprandial, Non-fasting, Triglyceride, Remnant cholesterol, Chinese subjects

\section{Introduction}

According to 2016 Chinese guideline for the management of dyslipidemia in adults, appropriate triglyceride (TG) level is defined as fasting TG $<1.7 \mathrm{mmol} / \mathrm{L}$, borderline elevation as $1.7 \mathrm{mmol} / \mathrm{L} \leq$ fasting $\mathrm{TG}<2.3 \mathrm{mmol} / \mathrm{L}$, and elevation as fasting $\mathrm{TG} \geq 2.3 \mathrm{mmol} / \mathrm{L}$ [1]. Actually, non-fasting or postprandial high TG (HTG) is also an

\footnotetext{
* Correspondence: feliuling@csu.edu.cn

All authors take responsibility for all aspects of the reliability and freedom from bias of the data presented and their discussed interpretation.

'Department of Cardiovascular Medicine, The Second Xiangya Hospital, Central South University, \#139 Middle Renmin Road, Changsha, Hunan 410011, People's Republic of China

${ }^{2}$ Research Institute of Blood Lipid and Atherosclerosis, Central South University, Changsha, Hunan 410011, People's Republic of China
}

independent risk factor of coronary heart disease (CHD) [2-4]. Non-fasting levels of blood lipids can be detected at anytime within $8 \mathrm{~h}$ after a daily meal according to individual's dietary habits or at appointed time-points after a high-fat meal with specified content. The former is similar to a random blood glucose measurement while the latter is analogue to oral glucose tolerance test [5]. According to the European joint consensus statement from the European Atherosclerosis Society (EAS) and the statement from the American Heart Association (AHA), non-fasting TG levels in patients whose fasting TG $<1.7 \mathrm{mmol} / \mathrm{L}$ would not be expected to raise above $2.0 \mathrm{mmol} / \mathrm{L}(175 \mathrm{mg} / \mathrm{dL})$ and $2.26 \mathrm{mmol} / \mathrm{L}(200 \mathrm{mg} / \mathrm{dL})$, respectively, after consuming a daily low fat meal before 
blood sampling [6, 7]. However, the cut-off point to diagnose non-fasting HTG is $1.98 \mathrm{mmol} / \mathrm{L}(175 \mathrm{mg} / \mathrm{dL})$ in middle aged and older apparently healthy American women basing on the Women's Health Study [8], which is very close to the optimal non-fasting TG cut-off point $(2.0 \mathrm{mmol} / \mathrm{L})$ in Japanese employees [9]. Until now, similar expert consensus or study is still rare in China. Recently, we determined a cut-off point for non-fasting TG level $2.02 \mathrm{mmol} / \mathrm{L}$ at $4 \mathrm{~h}$ after a daily breakfast in relation to fasting TG $\geq 1.7 \mathrm{mmol} / \mathrm{L}$ in 109 Chinese subjects with and without overweight [10], which is very close to the values recommended by the European joint consensus statement or reported by other scholars $[6,8,9]$. HTG indicates the increases in circulating triglyceride-rich lipoproteins (TRLs) and their remnants, remnant lipoproteins $[4,11,12]$. The latter is as atherogenic as lowdensity lipoprotein (LDL) [13, 14]. Level of remnant lipoprotein cholesterol (i.e. remnant cholesterol, RC) can be measured directly or estimated by a certain formula [15]. Numerous studies showed that estimated RC independently predicted the risk of CHD [16-18]. It is recommended that non-fasting $\mathrm{RC}$ level should not be above $0.9 \mathrm{mmol} / \mathrm{L}(35 \mathrm{mg} / \mathrm{dL})$ after a daily meal for individuals with fasting $\mathrm{RC}<0.8 \mathrm{mmol} / \mathrm{L}(30 \mathrm{mg} / \mathrm{dL})$ by the EAS [6], however, there is no similar recommendation in China.

Comparing with above studies about non-fasting cutoff point for TG elevation after a daily meal [7-9], those after a high-fat meal was scarce although fat tolerance test has been employed to evaluate postprandial HTG for a longer time since last century [19]. Only Mexican scholars had identified an optimal TG truncation of $3.17 \mathrm{mmol} / \mathrm{L}(280 \mathrm{mg} / \mathrm{dL})$ after a high-fat meal with 960 kcal through receiver operating characteristic (ROC) curve analysis [20]. We previously established an oral fat tolerance test with $800 \mathrm{kcal}$ and $56 \%$ fat content according to Chinese dietary habits, and observed the different changes in non-fasting TG levels between CHD patients and controls [21-23]. However, there was no study about non-fasting cut-off point for TG or RC elevation after a high-fat meal in Chinese yet.

In this study, we compared the changes in levels of TG and estimated RC between CHD patients and their controls after a high-fat meal, aimed to identify optimal non-fasting cut-off points of TG and RC in Chinese individuals after a high-fat meal in relation to fasting $\mathrm{TG} \geq$ $1.7 \mathrm{mmol} / \mathrm{L}$ and $\mathrm{RC} \geq 0.8 \mathrm{mmol} / \mathrm{L}$, respectively.

\section{Methods}

\section{Study subjects}

Ninety subjects, including 60 documented CHD patients (CHD group) and 30 non-CHD controls (CON group), were recruited in this study in the Department of Cardiovascular Medicine of the Second Xiangya Hospital,
Central South University. CHD was defined as a history of myocardial infarction and/or angiographically proven coronary atherosclerosis in patients with angina pectoris. Contemporaneous controls who had no clinical history and manifestation of $\mathrm{CHD}$ were classified into $\mathrm{CON}$ group [24].

All subjects were invited to filled out a questionnaire on medical history and use of medication before participant. No subjects had a history of diabetes, thyroid diseases, liver and kidney diseases, autoimmune disease, cancer or other severe medical illnesses, and no one took oral hypoglycaemic or hypolipidemic agents. The study was approved by the Ethics Committee of the Second Xiangya Hospital of Central South University and informed consent was gained from all participants.

\section{Oral high-fat tolerance test}

After at least $12 \mathrm{~h}$ of overnight fasting, all subjects were given a high-fat meal. The oral high-fat tolerance test was undertaken as described previously by a nutritionist [23]. The high-fat meal consisting of $800 \mathrm{kcal}$ with $50 \mathrm{~g}$ of fat ( $345 \mathrm{mg}$ of cholesterol), $28 \mathrm{~g}$ of protein, and $60 \mathrm{~g}$ of carbohydrate was given in Chinese traditional form, including vegetable blending oil, noodles, egg and pork floss. All subjects were requested to finish the meal in 15 min. During 6-h test, subjects were allowed to drink only water and prohibited to smoke, drink wine or eat any food. Strenuous exercises were not recommended, and only slow walking was allowed.

\section{Laboratory assays}

Blood samples were taken before and at 2, 4 and $6 \mathrm{~h}$ after the high-fat meal. All blood samples were centrifuged at $4{ }^{\circ} \mathrm{C} 3000 \mathrm{rpm}$ for $15 \mathrm{~min}$. Serum levels of total cholesterol (TC) and TG were measured by automated enzymatic assays, and that of high-density lipoprotein cholesterol (HDL-C) were measured by a commercially available direct method, on a HITACHI 7170A analyzer (Instrument Hitachi Ltd., Tokyo, Japan) by a laboratory technician who had no idea of this study. LDLcholesterol (LDL-C) level was calculated using the Friedewald formula: LDL-C $=$ TC - (HDL-C) - $(\mathrm{TG} / 2.2)$ when TG was $<4.5 \mathrm{mmol} / \mathrm{L}$, otherwise it was directly measured by chemical masking method. RC level was estimated by the following formula, $\mathrm{RC}=\mathrm{TC}-(\mathrm{HDL}-\mathrm{C})$ (LDL-C). Non-HDL-C = TC - (HDL-C). Fasting glucose level was measured using the glucose oxidase method.

\section{Statistical analysis}

Quantitative variables were expressed as mean \pm standard deviation (SD) unless were specifically explained, and qualitative variables were expressed as numbers and percentages. Differences between the intra- and intergroup means were analyzed by unpaired $t$-test or one- 
way analysis of variance. Categorical variables were compared using chi-squared statistic tests. The area under the curve (AUC) and the increment of AUC (iAUC), representing the increase in area after a high-fat meal above fasting levels of TG and RC, were estimated by trapezoid method. The optimal cut-off points for nonfasting TG and RC levels at $4 \mathrm{~h}$ were determined using ROC curve analysis. The rate of omissive diagnosis was defined as the percentage of normal fasting TG or RC in subjects with postprandial HTG or high RC (HRC). All statistical analyses were performed with SPSS version 25.0. All $P$ values were 2 -tailed, and $P<0.05$ was considered statistically significant.

\section{Results}

\section{Clinical characteristics and fasting blood lipids of two groups}

There were no significant difference in age, gender, body mass index (BMI), systolic or diastolic blood pressure, heart rate, percentage of overweight or smoking, and fasting glucose level between two groups. Levels of fasting TG, TC, LDL-C, non-HDL-C and RC were significantly higher while fasting HDL-C level was significantly lower in CHD group $(P<0.05$, Table 1$)$. Fasting TG elevation $(\geq 1.7 \mathrm{mmol} / \mathrm{L})$ was found in $42(70 \%) \mathrm{CHD}$ patients and $10(33.3 \%)$ controls, which was similar to that of fasting $\mathrm{RC}$ elevation $(\geq 0.8 \mathrm{mmol} / \mathrm{L})$ in each group (CHD: 68.3\%; CON: 30\%).

Table 1 Comparison of clinical features between two groups

\begin{tabular}{|c|c|c|c|}
\hline & $\begin{array}{l}\text { CHD } \\
(n=60)\end{array}$ & $\begin{array}{l}\text { CON } \\
(n=30)\end{array}$ & $P$ value \\
\hline Age (y) & $52.22 \pm 7.27$ & $49.40 \pm 11.25$ & NS \\
\hline Gender (M/F) & $42 / 18$ & $21 / 9$ & NS \\
\hline $\mathrm{BMI}\left(\mathrm{Kg} / \mathrm{m}^{2}\right)$ & $24.47 \pm 2.26$ & $23.91 \pm 2.75$ & NS \\
\hline Overweight [n(\%)] & $35(58.33)$ & $12(40.00)$ & NS \\
\hline Systolic pressure (mmHg) & $123.68 \pm 15.18$ & $120.43 \pm 16.92$ & NS \\
\hline Diastolic pressure $(\mathrm{mmHg})$ & $80.69 \pm 9.36$ & $78.48 \pm 10.92$ & NS \\
\hline Heart rate (bpm) & $77.12 \pm 8.13$ & $77.12 \pm 9.34$ & NS \\
\hline Current smoking [n(\%)] & $23(38.33)$ & $8(26.67)$ & NS \\
\hline Fasting glucose (mmol/L) & $5.22 \pm 0.69$ & $4.99 \pm 0.84$ & NS \\
\hline $\mathrm{TG}^{\mathrm{a}}(\mathrm{mmol} / \mathrm{L})$ & $2.36 \pm 1.20$ & $1.41 \pm 0.57$ & $<0.001$ \\
\hline $\mathrm{TC}(\mathrm{mmol} / \mathrm{L})$ & $5.16 \pm 0.78$ & $4.44 \pm 0.77$ & $<0.001$ \\
\hline $\mathrm{HDL}-\mathrm{C}^{\mathrm{a}}(\mathrm{mmol} / \mathrm{L})$ & $1.12 \pm 0.19$ & $1.26 \pm 0.31$ & $<0.05$ \\
\hline LDL-C (mmol/L) & $2.98 \pm 0.65$ & $2.54 \pm 0.69$ & $<0.01$ \\
\hline non-HDL-C (mmol/L) & $4.04 \pm 0.74$ & $3.18 \pm 0.71$ & $<0.001$ \\
\hline $\mathrm{RC}^{\mathrm{a}}(\mathrm{mmol} / \mathrm{L})$ & $1.06 \pm 0.53$ & $0.64 \pm 0.25$ & $<0.001$ \\
\hline
\end{tabular}

BMI Body mass index, bpm Beats per minute, TG Triglyceride, TC Total cholesterol, HDL-C High-density lipoprotein cholesterol, LDL-C Low-density lipoprotein cholesterol, non-HDL-C Non high-density lipoprotein cholesterol, $R C$ Remnant lipoprotein cholesterol, NS No significance

${ }^{a}$ logarithmic transformation for non-normal distribution
Postprandial changes in serum levels of blood lipids in two groups

Significant differences in blood lipids between CHD group and $\mathrm{CON}$ group were showed at most time points after a high-fat meal $(P<0.05$, Fig. 1a-f).

Although postprandial changes in levels of TC, HDL$\mathrm{C}$ and non-HDL-C were slight (Fig. 1a-c), postprandial decrease in LDL-C level was significant at $2 \mathrm{~h}$ and $4 \mathrm{~h}$ in both groups $(P<0.05$, Fig. $1 d)$. Postprandial LDL-C level at $6 \mathrm{~h}$ in $\mathrm{CON}$ group was still significantly lower than its fasting value $(P<0.05)$ while that in $\mathrm{CHD}$ group restored to near its fasting value (Fig. 1d).

Both TG and RC levels increased tremendously at all postprandial time points $(P<0.05)$ and peaked at $4 \mathrm{~h}$ after a high-fat meal in both groups (Fig. 1e \& f). AUC of TG or RC in CHD group was significantly higher than that in CON group $(P<0.05)$, however, only iAUC of TG in CHD group was significantly higher than that in CON group, but not $\mathrm{RC}(P<0.05$, Fig. $1 \mathrm{~g} \& \mathrm{~h})$.

\section{Comparisons of percentages of postprandial dyslipidemia according to cut-off points recommended by the EAS between two groups}

Postprandial levels of TG and RC in this study were initially evaluated according to the non-fasting cut-off points after a daily meal recommended by the EAS. Postprandial percentages of HTG and HRC in CHD group were prominently higher than those in CON group, respectively $(P<$ 0.05). Percentage of HTG or HRC in postprandial state at any time point was enormously higher than that in fasting state in each group $(P<0.05)$. The highest percentage of HTG or HRC in each group was found at $4 \mathrm{~h}$, which was more than $90 \%$ in CHD group and reached $60 \%$ in CON group (Fig. 2a \& b).

Furthermore, when the time of peak level of TG or RC was analyzed, it was found mainly at $4 \mathrm{~h}$ in CHD group but nearly equally at $2 \mathrm{~h}$ and $4 \mathrm{~h}$ in CON group. There was significant difference in percentage of TG reaching the peak level at $2 \mathrm{~h}$ or $4 \mathrm{~h}$ between two groups $(P<0.05$, Fig. $2 \mathrm{c} \& \mathrm{~d})$.

\section{Comparisons of percentages of postprandial dyslipidemia according to new cut-off points determined by ROC curve analysis between two groups}

ROC curve analysis was performed and Youden's index was calculated according to the sensitivity and specificity of each possible cut-off point in the statistical results. The optimal cut-off point for TG at $4 \mathrm{~h}$ to predict HTG in relation to fasting TG $\geq 1.7 \mathrm{mmol} / \mathrm{L}$ was $3.12 \mathrm{mmol} / \mathrm{L}$ (sensitivity 86.5\%, specificity $86.8 \%$, and AUC 0.917), and that for $\mathrm{RC}$ at $4 \mathrm{~h}$ to predict $\mathrm{HRC}$ in relation to fasting $\mathrm{RC} \geq 0.8 \mathrm{mmol} / \mathrm{L}$ was $1.36 \mathrm{mmol} / \mathrm{L}$ (sensitivity $82 \%$, specificity $82.5 \%$, and AUC 0.878) (Fig. 3a \& b).

According to the new cut-off points, the percentages of postprandial HTG and HRC decreased obviously. 


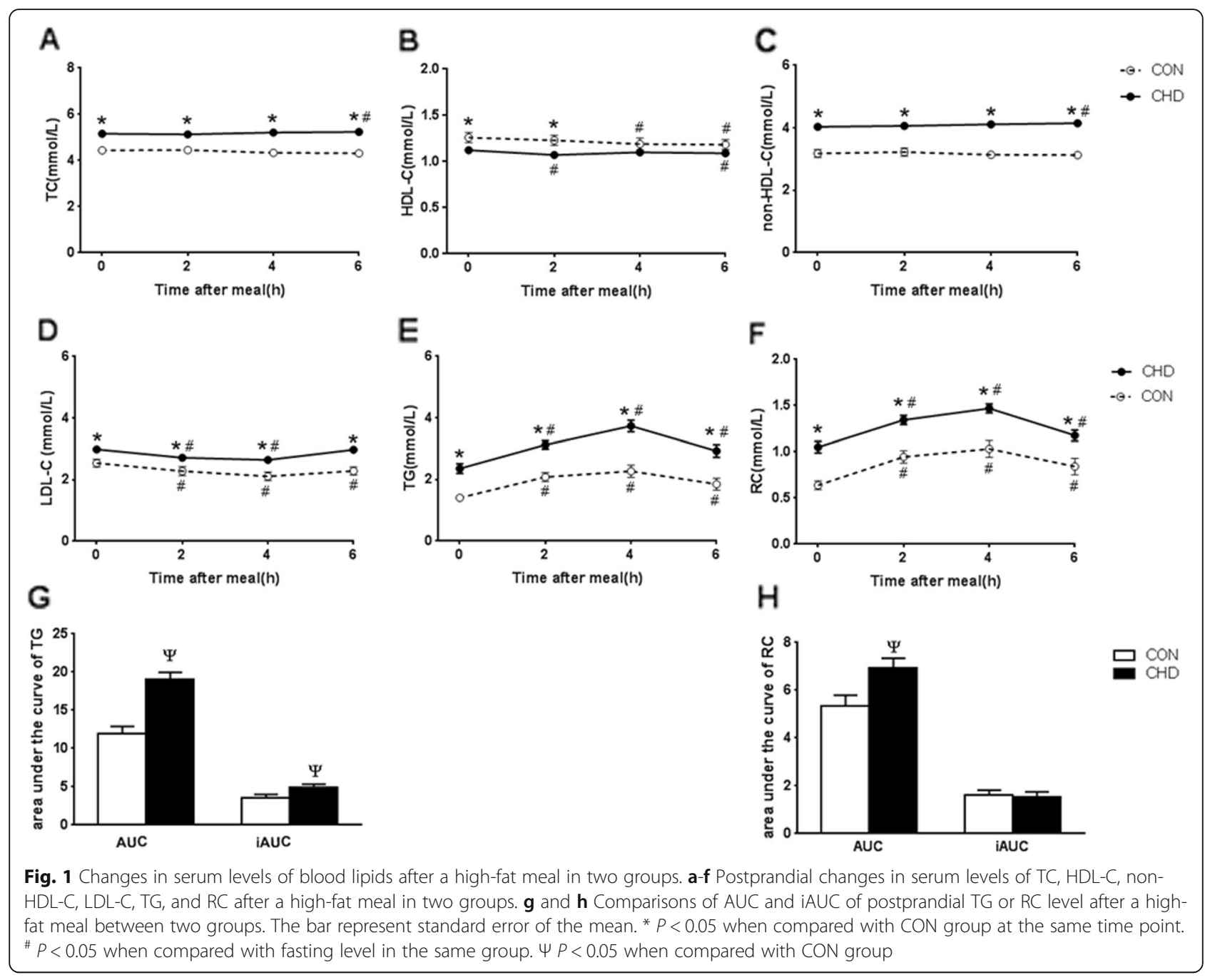

They were no more than $70 \%$ in CHD group while less than $30 \%$ in CON group. Significant difference in percentage of postprandial HTG or HRC between two groups was found at $2 \mathrm{~h}$ and $4 \mathrm{~h}(P<0.05)$. Furthermore, the highest percentage of postprandial hyperlipidemia, i.e. HTG or HRC, was still found at $4 \mathrm{~h}$ in both groups $(P<0.05$, Fig. $3 c$ \& d $)$.

When postprandial HTG or HRC was defined as the maximal postprandial TG $(\mathrm{pTGm}) \geq 2.0 \mathrm{mmol} / \mathrm{L}$ or $\mathrm{RC}(\mathrm{pRCm}) \geq 0.9 \mathrm{mmol} / \mathrm{L}$, postprandial $\mathrm{HTG}$ or HRC was found in about two-thirds controls and near $100 \%$ patients with CHD no matter fasting TG or RC level was borderline-high or not. When postprandial HTG or HRC was defined as $\mathrm{pTGm} \geq 3.12 \mathrm{mmol} / \mathrm{L}$ or $\mathrm{pRCm} \geq 1.36 \mathrm{mmol} / \mathrm{L}$, the percentage of postprandial HTG or HRC obviously decreased in each group, which mainly occurred in the subjects with appropriate fasting TG or RC level, while that changed mildly in those with fasting HTG or HRC, especially in CHD group (Fig. 3e \& f).
For subjects with appropriate fasting TG or RC level, the diagnosis of postprandial HTG or HRC could be omitted without detecting of non-fasting level after a high-fat meal. When the EAS cut-off points of postprandial hyperlipidemia were utilized, the omissive diagnosis rates of postprandial HTG and HRC were near 30\% in CHD group and about $50 \%$ in CON group, respectively. When the new cut-off points were utilized, they obviously decreased to $\leq 13 \%$ in CHD group and $33.3 \%$ in CON group (Fig. $3 g$ \& h).

\section{Discussion}

In this study, prominent non-fasting hyperlipidemia, presenting as elevated levels of TG and RC, was found in CHD patients after a high-fat meal, especially at $4 \mathrm{~h}$. Moreover, non-fasting cut-off points of HTG and HRC after a high-fat meal were firstly determined by ROC curve analysis in Chinese subjects, and they were obviously higher than those after a daily meal recommended by European consensus statement [6], however, the cut- 

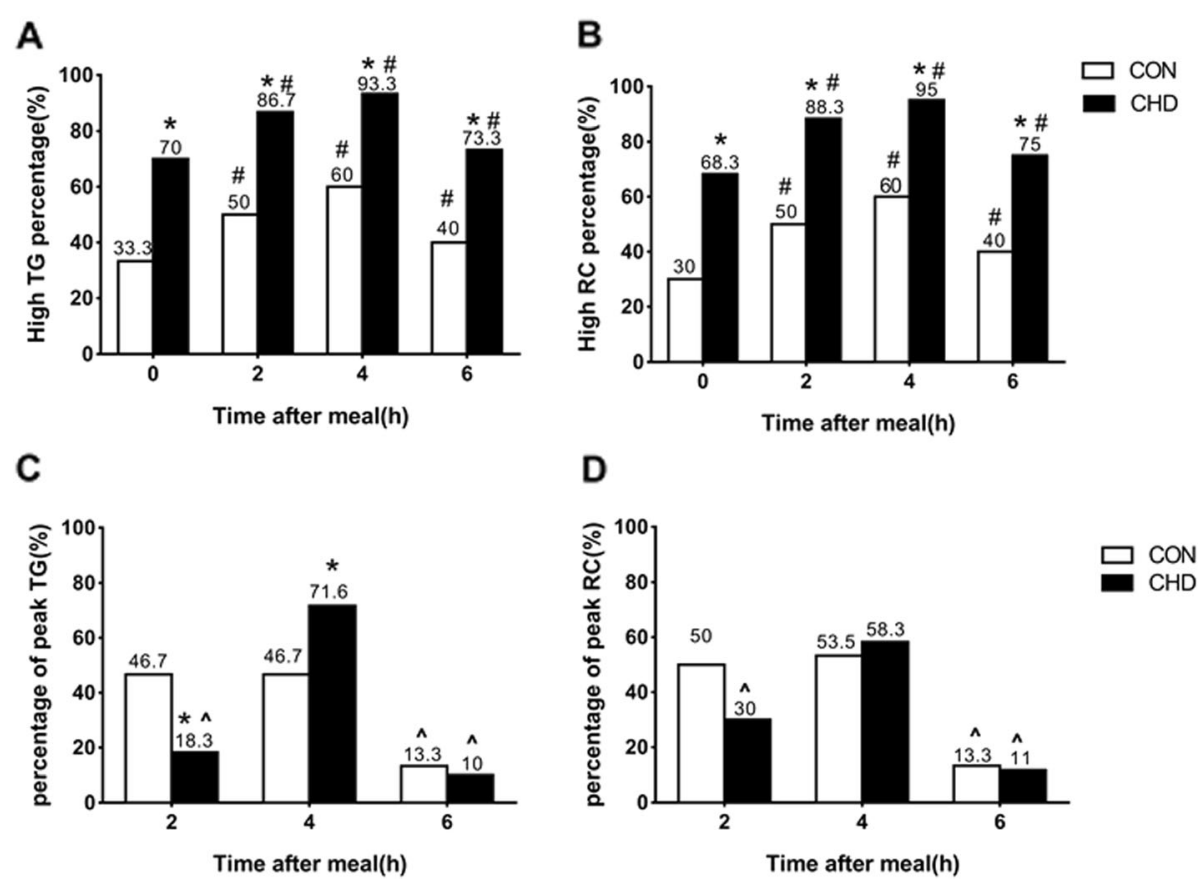

Fig. 2 Comparisons of the fasting and postprandial percentages of HTG and HRC between two groups according to the criteria recommended by the European Atherosclerosis Society (EAS) after a daily meal. $\mathbf{a}$ and $\mathbf{b}$ The percentages of HTG or HRC according to fasting and postprandially TG or RC level, respectively. $\mathbf{c}$ and $\mathbf{d}$ Comparisons of percentages of subjects reaching the peak value of TG or RC level at different time point after a high-fat meal. * $P<0.05$ when compared with CON group at the same time point. ${ }^{\#} P<0.05$ when compared with the value in fasting state in the same group. ${ }^{\wedge} P<0.05$ when compared with the postprandial percentage at $4 \mathrm{~h}$ in the same group

off point of non-fasting HTG in our study was very similar to that in another Mexican research [20]. These results not only supported increased synthesis and/or reduced elimination of TRLs and their remnants in Chinese CHD patients after a high-fat meal, but also indicated the difference in non-fasting cut-off points of HTG and HRC between a high-fat meal and a daily meal.

Postprandial state plays a critical role in atherogenesis [25-27], which was supported by our finding that AUC of TG or RC in CHD group was significantly higher than that in CON group, although there was no significant difference in iAUC of RC between two groups. When compared with iAUC that represents postprandial increment of TG or RC after a high-fat meal, AUC seems to be a better index to reflect the sustained stimulus and even damages of TRLs and their remnants to artery walls during postprandial period, because it not only reflects the fasting levels of blood lipids but also covers their non-fasting increments and duration after a high-fat meal. However, non-fasting changes in TC, HDL-C and non-HDL-C levels seemed to be negligible when compared with those in TG and RC after a high-fat meal, although postprandial LDL$C$ level showed a significant decrease in this study. In fact, it is possible that the change of LDL-C could become insignificant with the increase in samples size [28].
For there were no recommendation about cut-off points of postprandial HTG and HRC in Chinese subjects, postprandial TG and RC elevation in this study were initially evaluated according to the non-fasting cutoff points after a daily meal recommended by the EAS [6]. Non-fasting HTG was found in 73.3-93.3\% CHD patients and about $50 \%$ in the controls. The percentages of non-fasting HRC in two groups were very similar to those of non-fasting HTG because RC was estimated by formula. Under this condition, the proportion of socalled postprandial hyperlipidemia was too excessive to differentiate postprandial TRL metabolic abnormalities, especially in CHD patients, from physiological TG reaction to a high-fat meal, mainly in the controls [29]. We previously observed that postprandial TG elevation after a high-fat meal was greater than that after a low-fat meal in Chinese subjects [22], suggesting that it would be inappropriate to evaluate non-fasting levels of TG and RC in Chinese subjects after a high-fat meal according to the cut-off points after a daily meal recommended by the EAS .

In our study, the peak levels of TG and RC in CHD group predominantly emerged at $4 \mathrm{~h}$ in this study, while those in CON group were approximately in uniform distribution at $2 \mathrm{~h}$ and $4 \mathrm{~h}$, indicating abnormal anabolism and/or catabolism of TRLs and their remnants in CHD 

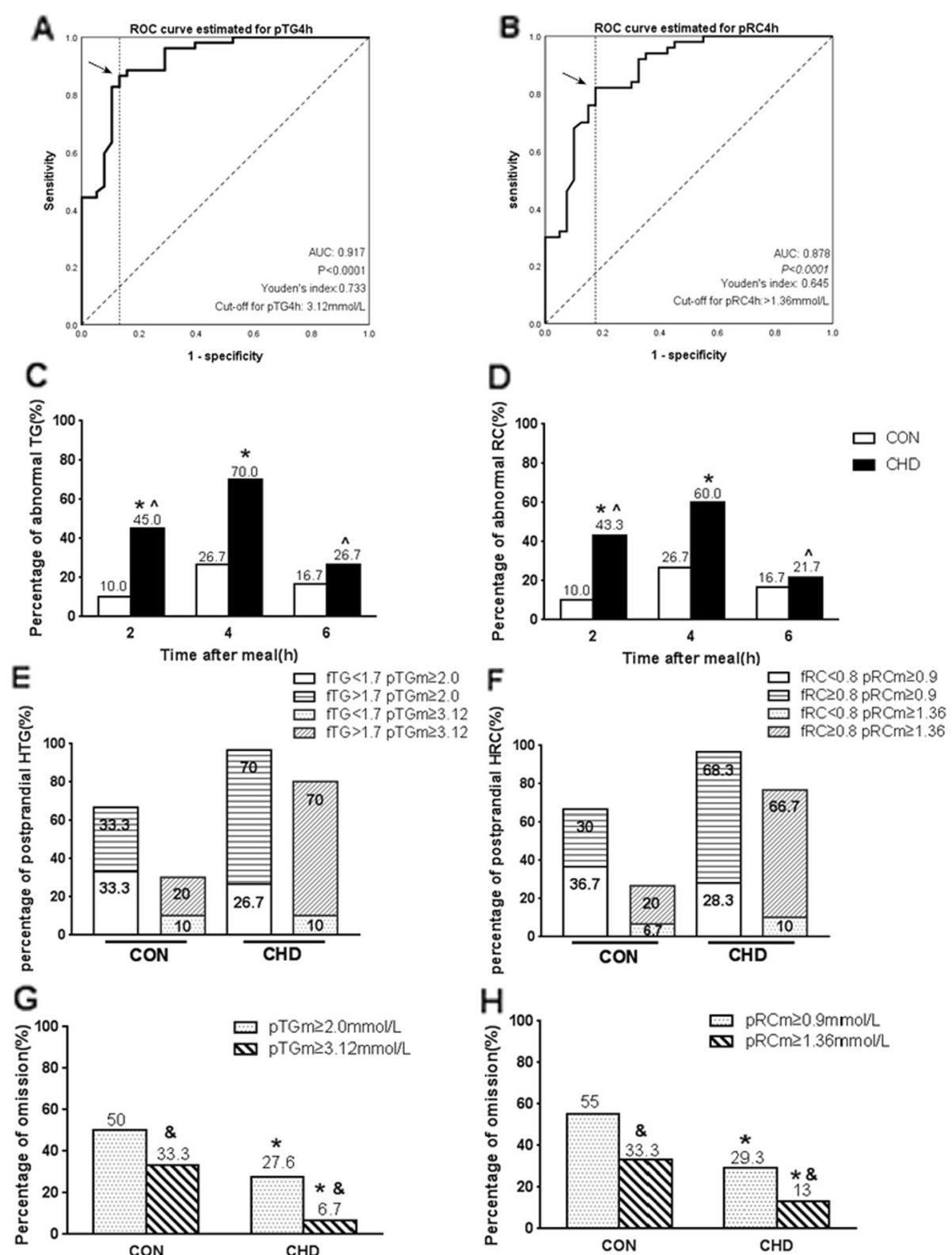

Fig. 3 Comparisons of the fasting and postprandial percentages of HTG and HRC between two groups according to the cut-off points via ROC analysis after a high-fat meal. $\mathbf{a}$ and $\mathbf{b}$ ROC analysis and Youden's index determined a cut-off point for postprandial TG or RC level at $4 \mathrm{~h}$ (pTG4h or pRC4h) after a high-fat meal, the cut-off point was indicated by the solid arrow. $\mathbf{c}$ and $\mathbf{d}$ Comparisons of postprandial percentages of HTG or HRC at different time point according to new cut-off points. e and $\mathbf{f}$ Comparison of percentage of postprandial HTG or HRC according to different postprandial criteria in two group. $\mathbf{g}$ and $\mathbf{h}$ Comparison of omissive diagnosis rates according to different postprandial criteria of HTG and HRC between two groups. ${ }^{*} P<0.05$ when compared with CON group according to a same criteria. ${ }^{\wedge} P<0.05$ when compared with the percentage at $4 \mathrm{~h}$ in the same group. ${ }^{\&} P<0.05$ when compared with omissive diagnosis rates according to EAS criteria in the same group

patients. In view of a majority of subjects reaching the peak level of either TG or RC at $4 \mathrm{~h}$ after a high-fat meal, along with our previous finding that the optimal time point to evaluate postprandial TG elevation could be at $4 \mathrm{~h}$ after a high-fat meal with $800 \mathrm{kcal}$ in Chinese subjects [30], we analyzed the cut-off points of nonfasting TG and RC elevation at this time point in the subsequent ROC curve analysis.
ROC curve analysis had been employed to determine optimal cut-off points for non-fasting high TG not only after a daily meal in the Women's Health Study [8], but also after a high-fat meal in another study in Mexican adults [20]. In this study, the cut-off point of postprandial high TG was $3.12 \mathrm{mmol} / \mathrm{L}$ after a high-fat meal with $800 \mathrm{kcal}$ in Chinese subjects, obviously higher than 2.0 $\mathrm{mmol} / \mathrm{L}$ that is recommended by the EAS after a daily 
meal [6], while was very close to another cut-off point of TG, $3.16 \mathrm{mmol} / \mathrm{L}$, after a high-fat meal with $960 \mathrm{kcal}$ in Mexican adults [20]. The similarity between two cut-off points, $3.12 \mathrm{mmol} / \mathrm{L}$ and $3.16 \mathrm{mmol} / \mathrm{L}$, for non-fasting high TG might not be simply attributed to a coincidence, but result from similar fat contents, $50 \mathrm{~g}$ vs. $52 \mathrm{~g}$, of two high-fat meals in our study and the Mexican study. It suggests that high-fat meals with similar total calorie and fat content may share similar cut-off points, although the race of the subjects, dietary habits, time points for lipid testing and statistical methods were different between two studies.

Besides TG, we also analyzed RC in the postprandial state through $\mathrm{ROC}$ curve, as $\mathrm{RC}$ is an independent risk factor for cardiovascular diseases [13, 14]. A cut-off point of $\mathrm{RC}, 1.36 \mathrm{mmol} / \mathrm{L}$, was determined to discriminate fasting $\mathrm{RC}$ elevation and much higher than that recommended by the EAS, $0.9 \mathrm{mmol} / \mathrm{L}$. It indicated that for Chinese patients taking a high-fat meal with $800 \mathrm{kcal}$, a specific cut-off point of RC, but not that one after a daily meal [6], was needed.

When the new cut-off points determined by ROC curve analysis were used for evaluation, the percentage of postprandial HTG or HRC in each group was relatively lower than that evaluated by the cut-off points recommended by the EAS, however, the difference in multiples of the proportion of postprandial HTG or HRC between two groups increased obviously at $2 \mathrm{~h}$ and $4 \mathrm{~h}$. For example, the proportion of postprandial HTG in CHD group was more than two times at $4 \mathrm{~h}$ as much as that in CON group, which was very similar to the condition in the fasting state. It indicated new cut-off points could be more helpful to differentiate the controls from $\mathrm{CHD}$ patients. Indeed, there were some individuals in CON group with risk factors, such as smoking [31, 32], overweight [33] and fasting TG or RC elevation [4], thus it was acceptable that HTG was found in $26.7 \%$ of the controls at $4 \mathrm{~h}$ after a high-fat meal.

It was worth noting that postprandial HTG or HRC was found in part of subjects with appropriate fasting TG or RC level when the maximal postprandial TG or RC level were evaluated, indicating that quite a part of subjects would be missed if postprandial blood lipids were not assessed. For this reason, omission diagnostic rates were analyzed according to different cut-off points. Interestingly, the omission diagnostic rates of postprandial HTG and HRC determined according to the new cut-off points were lower than that according to the EAS cut-off points in each group, suggesting that it is necessary to determine specific diagnose thresholds of postprandial hyperlipidemia for different population after a certain high-fat meal.

This study is associated with several limitations. First, the sample size in this study was small. Second, both
LDL-C and RC were calculated by formulae, which may cause deviation with those directly measured [34].

\section{Conclusion}

In conclusion, the cut-off points of postprandial HTG and HRC corresponding to fasting HTG and HRC were determined firstly in Chinese subjects after a high-fat meal and were found to be higher than those recommended by the EAS after a daily meal.

\begin{abstract}
Abbreviations
AHA: American Heart Association; AUC: Area under the curve; CHD: Coronary heart disease; CON: Non-CHD controls; EAS: European Atherosclerosis Society; HDL-C: High-density lipoprotein cholesterol; HRC: High remnant cholesterol; HTG: High triglyceride; iAUC: Increment of AUC; LDL: Low-density lipoprotein; LDL-C: Low-density lipoprotein cholesterol; Non-HDL-C: Nonhigh-density lipoprotein cholesterol; pRC: Postprandial RC;

pRC4h: Postprandial RC at $4 \mathrm{~h}$ after meal; pRCm: The maximal postprandial RC; pTG: Postprandial TG; pTG4h: Postprandial TG at $4 \mathrm{~h}$ after meal; pTGm: The maximal postprandial TG; RC: Remnant cholesterol; ROC curve: Receiver operating characteristic; TC: Total cholesterol; TG: Triglyceride; TRLs: Triglyceride-rich lipoproteins
\end{abstract}

\section{Acknowledgments}

Not applicable.

\section{Authors' contributions}

$J X$ and LL carried out the experimental work and the data collection and interpretation. YQ C, SP Z and LL participated in the design and coordination of experimental work, and acquisition of data. LL and SP Z participated in the study design, data collection, analysis of data and preparation of the manuscript. JX and LL carried out the study design, the analysis and interpretation of data and drafted the manuscript. All authors read and approved the final manuscript.

\section{Funding}

The study was supported by National Natural Science Foundation of China [grant numbers 81270956, 81470577].

\section{Availability of data and materials}

The datasets analyzed during the current study are available from the corresponding author on reasonable request.

\section{Ethics approval and consent to participate}

The study was approved by the Ethics Committee of the Second Xiangya Hospital of Central South University and informed consent was gained from all participants.

\section{Consent for publication}

Not applicable.

\section{Competing interests}

The authors declare that they have no competing interests.

Received: 15 September 2019 Accepted: 7 November 2019

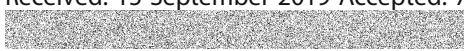

\section{References}

1. Hu DY. New guidelines and evidence for the prevention and treatment of dyslipidemia and atherosclerotic cardiovascular disease in China. Zhonghua Xin Xue Guan Bing Za Zhi. 2016;44(44):826-7.

2. Kolovou GD, et al. Postprandial hypertriglyceridaemia revisited in the era of non-fasting lipid profile testing: a 2019 expert panel statement, main text. Curr Vasc Pharmacol. 2019;17(5):498-514.

3. Giuseppe DP, et al. Effects of a diet naturally rich in polyphenols on lipid composition of postprandial lipoproteins in high cardiometabolic risk individuals: an ancillary analysis of a randomized controlled trial. Eur J Clin Nutr. 2019. https://doi.org/10.1038/s41430-019-0459-0. 
4. Kolovou G, Ooi TC. Postprandial lipaemia and vascular disease. Curr Opin Cardiol. 2013;28(4):446-51.

5. Kolovou GD, et al. Assessment and clinical relevance of non-fasting and postprandial triglycerides: an expert panel statement. Curr Vasc Pharmacol. 2011;9(3):258.

6. Nordestgaard BG, et al. Fasting is not routinely required for determination of a lipid profile: clinical and laboratory implications including flagging at desirable concentration cut-points - a joint consensus statement from the European Atherosclerosis Society and European Federation of Clinical Chemistry and Laboratory Medicine. Eur Heart J. 2016;37(25):1944-58.

7. Miller M, et al. Triglycerides and cardiovascular disease. Circulation. 2011; 123(20):2292-333.

8. White KT, et al. Identifying an optimal cutpoint for the diagnosis of hypertriglyceridemia in the nonfasting state. Clin Chem. 2015;61(9):1156-63.

9. Takahara $\mathrm{M}$, et al. Evaluation of hypertriglyceridemia using non-fasting health checkup data in a Japanese population. J Atheroscler Thromb. 2013; 20(8):661-9.

10. Tian F, et al. Changes in non-fasting concentrations of blood lipids after a daily Chinese breakfast in overweight subjects without fasting hypertriglyceridemia. Clin Chim Acta. 2019;490:147-53.

11. Fernandez-Arroyo S, et al. Effect of Vitamin D3 on the postprandial lipid profile in obese patients: a non-targeted lipidomics study. Nutrients. 2019; 11(5):1194.

12. Zhang $M$, et al. Indispensable role of lipoprotein bound-ApoE in adipogenesis and endocytosis induced by postprandial TRL. Biochem Biophys Res Commun. 2017;493(1):298-305

13. Ginsberg HN, Illingworth DR. Postprandial dyslipidemia: an atherogenic disorder common in patients with diabetes mellitus. Am J Cardiol. 2001; 88(6A): $9 \mathrm{H}-15 \mathrm{H}$.

14. Bansal $S$, et al. Fasting compared with nonfasting triglycerides and risk of cardiovascular events in women. JAMA. 2007;298(3):309-16.

15. Varbo A, et al. Elevated remnant cholesterol causes both low-grade inflammation and ischemic heart disease, whereas elevated low-density lipoprotein cholesterol causes ischemic heart disease without inflammation. Circulation. 2013;128(12):1298-309.

16. Kai-juan W, et al. Correlation of remnant lipoprotein-cholesterol (RemL-C) with the development of coronary heart disease (CHD) in the patients achieving LDL-cholesterol goals. Chin J Clin Lab Sci. 2016;34(08):601-4.

17. McNamara JR, et al. Remnant-like particle (RLP) cholesterol is an independent cardiovascular disease risk factor in women: results from the Framingham Heart study. Atherosclerosis. 2001;154(1):229-36.

18. Peng J, et al. Hypertriglyceridemia and atherosclerosis. Lipids Health Dis. 2017;16(1):233

19. Vogel RA, Corretti MC, Plotnick GD. Effect of a single high-fat meal on endothelial function in healthy subjects. Am J Cardiol. 1997;79(3):350-4.

20. Sevilla-González MDR, et al. Identification of a threshold to discriminate fasting hypertriglyceridemia with postprandial values. Lipids Health Dis. 2018;17(1):156

21. Zhao SP, et al. Impairment of endothelial function after a high-fat meal in patients with coronary artery disease. Coron Artery Dis. 2001;12(7):561-5.

22. Huili Z, et al. Postprandial Lipoprote in Metabolism and Impairment of Endothelial Function in Coronary Heart Disease. Chin Circ J. 2001;16(01):2527.

23. Zhao SP, et al. Xuezhikang, an extract of cholestin, protects endothelial function through antiinflammatory and lipid-lowering mechanisms in patients with coronary heart disease. Circulation. 2004;110(8):915-20

24. Hu D, Yang Y, Peng D. Increased sortilin and its independent effect on circulating proprotein convertase subtilisin/kexin type 9 (PCSK9) in statinnaive patients with coronary artery disease. Int J Cardiol. 2017;227:61-5.

25. Zilversmit DB. Atherogenesis: a postprandial phenomenon. Circulation. 1979; 60(3):473-85.

26. Kakuda $\mathrm{H}$, et al. The effect of anagliptin treatment on glucose metabolism and lipid metabolism, and oxidative stress in fasting and postprandial states using a test meal in Japanese men with type 2 diabetes. Endocrine. 2015; 48(3):1005-9.

27. Mooij HL, et al. Ext1 heterozygosity causes a modest effect on postprandial lipid clearance in humans. J Lipid Res. 2015;56(3):665-73.

28. Langsted A, Freiberg JJ, Nordestgaard BG. Fasting and nonfasting lipid levels: influence of normal food intake on lipids, lipoproteins, apolipoproteins, and cardiovascular risk prediction. Circulation. 2008;118(20): 2047-56.
29. Lina W. Metabolic Abnormality of Triglyceride-Rich Lipoproteins and Atherosclerosis. Advances in Cardiovascular Diseases. 2003;24(05):379-382.

30. Ling L, et al. Optimal time point to measure postprandial hypertriglyceridemia after a high2fat meal. Chin J Endocrinol Metab. 2004; 20(01):23-25.

31. Mero N, et al. Postprandial elevation of ApoB-48-containing triglyceride-rich particles and retinyl esters in normolipemic males who smoke. Arterioscler Thromb Vasc Biol. 1997;17(10):2096-102.

32. Axelsen M, et al. Lipid intolerance in smokers. J Intern Med. 1995; 237(5):449-55.

33. Blackburn $P$, et al. Postprandial hyperlipidemia: another correlate of the "hypertriglyceridemic waist" phenotype in men. Atherosclerosis. 2003; 171(2):327-36.

34. Lin Q, et al. Comparison of non-fasting LDL-C levels calculated by Friedewald formula with those directly measured in Chinese patients with coronary heart disease after a daily breakfast. Clin Chim Acta. 2019;495:399-405.

\section{Publisher's Note}

Springer Nature remains neutral with regard to jurisdictional claims in published maps and institutional affiliations.
Ready to submit your research? Choose BMC and benefit from:

- fast, convenient online submission

- thorough peer review by experienced researchers in your field

- rapid publication on acceptance

- support for research data, including large and complex data types

- gold Open Access which fosters wider collaboration and increased citations

- maximum visibility for your research: over $100 \mathrm{M}$ website views per year

At BMC, research is always in progress.

Learn more biomedcentral.com/submissions 